\title{
Bremsstrahlung energy loss of electrons passing through a plasma
}

\author{
E. Haug \\ Institut für Astronomie und Astrophysik, Universität Tübingen, 72076 Tübingen, Auf der Morgenstelle 10, Germany \\ e-mail: haug@tat.physik.uni-tuebingen.de
}

Received 3 March 2004 / Accepted 25 May 2004

\begin{abstract}
The average energy loss due to bremsstrahlung emission of electrons passing through a plasma is calculated. In particular, the contribution of electron-electron bremsstrahlung is specified. The bremsstrahlung losses are compared with the energy loss by collisions with ambient electrons. Above electron energies of $510 \mathrm{MeV}$ the energy loss due to bremsstrahlung predominates.
\end{abstract}

Key words. radiation mechanisms: nonthermal

\section{Introduction}

Electrons passing through a plasma lose energy by collisions with ambient particles and by emission of bremsstrahlung. Whereas in most cases the energy loss of electrons is almost entirely due to collisions, the emission of bremsstrahlung photons with energies of the order of magnitude of the electron kinetic energy will be predominant at very high electron energies, as was already pointed out by Heitler (1954). The emitted radiation is composed of electron-nucleus (e-n) and of electron-electron (e-e) bremsstrahlung. The calculation of the energy loss by e-n bremsstrahlung causes no difficulties since astrophysical plasmas are composed mainly of hydrogen and helium with minor admixtures of heavier elements. Therefore the relativistic cross section derived by Bethe \& Heitler (1934) in Born approximation is sufficient. In addition there exist accurate approximations to this cross section (Haug 1997). On the other hand, the evaluation of the e-e energy loss is complicated by the lengthy formulae for the cross section (Haug 1998). It has been performed in a rough approximation by substituting the factor $Z(Z+1)$ for the factor $Z^{2}$ in the e-n bremsstrahlung cross section, where $Z$ is the atomic number of the target nucleus. Moreover, the extreme relativistic approximation of the bremsstrahlung cross section is used (Blumenthal \& Gould 1970). The relative error of this procedure is rather high, $\approx 28 \%$ at electron energy $E=100 \mathrm{keV}$, and still $\approx 10 \%$ at $E=3.5 \mathrm{MeV}$. The aim of the present note is to provide simple formulae which lead to accurate values of the energy loss due to bremsstrahlung. The plasma is assumed to be sufficiently thin and hot so that the weak shielding and complete ionization approximations can be applied.

\section{Bremsstrahlung energy loss rate}

The average energy loss per unit time by bremsstrahlung emission of an electron of kinetic energy $E=(\epsilon-1) m c^{2}$ in a plasma is given by

$\frac{\mathrm{d} E}{\mathrm{~d} t}=-N m c^{2} v \int_{0}^{\epsilon-1} k \frac{\mathrm{d} \sigma}{\mathrm{d} k} \mathrm{~d} k$,

where $N$ denotes the number density of the target particles, $v$ the electron velocity, $m c^{2}$ the electron rest energy, $k=h v / m c^{2}$ the photon energy in units of $m c^{2}$, and $\mathrm{d} \sigma / \mathrm{d} k$ the bremsstrahlung cross section. The electron velocity $v$ can be expressed by the dimensionless energy $\epsilon$ and the momentum $p=\sqrt{\epsilon^{2}-1}, v=(p / \epsilon) c$. For e-n bremsstrahlung in a plasma with number density $N_{Z}$ of nuclei with atomic numbers $Z$, the cross section in Born approximation is proportional to the sum

$S=\sum_{Z} Z^{2} N_{Z}=N_{\mathrm{H}}\left\{1+\sum_{Z \geq 2}\left(N_{Z} / N_{\mathrm{H}}\right) Z^{2}\right\}$

$N_{\mathrm{H}}$ is the number density of hydrogen and $N_{Z} / N_{\mathrm{H}}$ the abundance relative to hydrogen of the elements with $Z \geq 2$. Using the cross section in Born approximation the integral 
in (1) can be carried out analytically (Heitler 1954). The nonrelativistic and extreme relativistic limits of this expression have the form

$\int_{0}^{\epsilon-1} k \frac{\mathrm{d} \sigma}{\mathrm{d} k} \mathrm{~d} k \approx \frac{16}{3} \alpha r_{0}^{2} Z^{2}\left(1+\frac{p^{2}}{3}+\frac{89}{1800} p^{4}-\frac{85}{1764} p^{6}\right)$,

$p^{2} \ll 1$,

and

$\int_{0}^{\epsilon-1} k \frac{\mathrm{d} \sigma}{\mathrm{d} k} \mathrm{~d} k \approx 4 \alpha r_{0}^{2} Z^{2} \epsilon\left\{\ln (2 \epsilon)-\frac{1}{3}\right\}, \quad \epsilon \gg 1$,

respectively, where $\alpha \approx 1 / 137$ is the fine-structure constant and $r_{0}$ the classical electron radius. An accurate approximation, which is valid for the whole energy range, can be derived from these limits, thus avoiding the calculation of the dilogarithm occurring in the exact formula. It has the form

$$
\begin{aligned}
\int_{0}^{\epsilon-1} k \frac{\mathrm{d} \sigma}{\mathrm{d} k} \mathrm{~d} k \approx & 8 \alpha r_{0}^{2} Z^{2} \frac{\epsilon^{3}}{\epsilon^{2}+p^{2}}\left\{\frac{\epsilon}{p} \ln (\epsilon+p)-\frac{1}{3}\right. \\
& \left.+\frac{p^{2}}{\epsilon^{6}}\left(\frac{2}{9} \epsilon^{2}-\frac{19}{675} \epsilon p^{2}-0.06 p^{4} / \epsilon\right)\right\} .
\end{aligned}
$$

Expanding the right-hand side of Eq. (5) into powers of $p^{2}$, it agrees with Eq. (3), and for $\epsilon \gg 1$ the leading terms of Eq. (5) are equal to the high-energy approximation (4) of Heitler's formula. The relative error of Eq. (5) is less than $0.54 \%$ throughout and less than $0.1 \%$ for electron energies $E<370 \mathrm{keV}$ and $E>5.8 \mathrm{MeV}$. At low energies $E$ one has to bear in mind that the true bremsstrahlung energy losses are somewhat higher due to the Coulomb correction of the cross section. In this region, however, the collision losses are dominating (see below) so that this does not matter.

For e-e bremsstrahlung the integral over $k$ occurring in Eq. (1) has to be calculated numerically. Here the upper integration limit is $k_{\max }=(\epsilon-1) /(\epsilon-p+1)$. Using the differential cross section including the Coulomb correction factor (Haug 1975) the cross section

$\Phi_{\mathrm{rad}}^{\mathrm{ee}}=\frac{1}{\epsilon-1} \int_{0}^{k_{\max }} k \frac{\mathrm{d} \sigma}{\mathrm{d} k} \mathrm{~d} k$

has been calculated. The resulting values were fitted to the following analytical expressions:

$$
\begin{aligned}
\Phi_{\mathrm{rad}}^{\mathrm{ee}} \approx & \alpha r_{0}^{2} \frac{\epsilon+1}{\epsilon}\left(0.6664+43.935 p-2.272 p^{2}-3.055 p^{3}\right) \\
& \times\left(1-\mathrm{e}^{2 \pi \alpha \epsilon / p}\right), \quad E \leq 100 \mathrm{keV}, \\
\Phi_{\mathrm{rad}}^{\mathrm{ee}} \approx & \alpha r_{0}^{2} \frac{\epsilon+1}{\epsilon}\left(0.5754+1.4492 \epsilon-0.0665 \epsilon^{2}\right), \\
& 100 \mathrm{keV} \leq E \leq 1 \mathrm{MeV}, \\
\Phi_{\mathrm{rad}}^{\mathrm{ee}} \approx & \alpha r_{0}^{2}(\epsilon+1) \frac{4.181 \epsilon \ln (\epsilon+p)-2.676 \epsilon-2.256}{\epsilon^{2}+1.022 \epsilon-3.871}, \\
& 1 \mathrm{MeV} \leq E \leq 500 \mathrm{MeV} .
\end{aligned}
$$

For $E>500 \mathrm{MeV}$ the formula

$$
\Phi_{\mathrm{rad}}^{\mathrm{ee}} \approx 4 \alpha r_{0}^{2}\left\{\ln (2 \epsilon)-\frac{1}{3}\right\}
$$

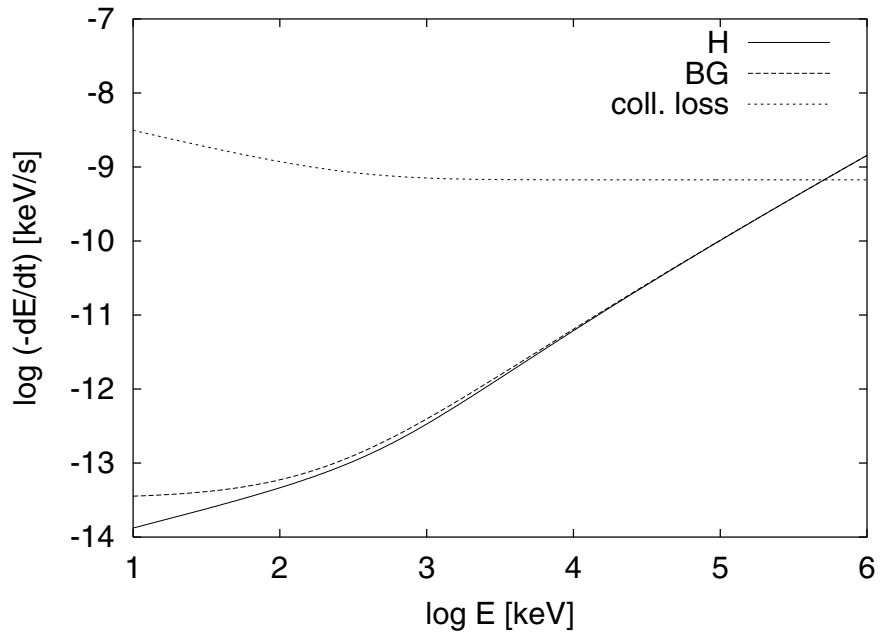

Fig. 1. Energy loss rate of electrons with kinetic energy $E$ due to bremsstrahlung according to Eq. (9) (H) and according to the approximation of Blumenthal and Gould (BG), compared with the energy loss rate by e-e collisions (coll. loss) in a completely ionized plasma with hydrogen number density $N_{\mathrm{H}}=1 \mathrm{~cm}^{-3}$ and ratio $N_{\mathrm{He}} / N_{\mathrm{H}}=0.1$.

can be applied which follows from the cross section for ultrarelativistic electrons (Baĭer et al. 1967).

Assuming a completely ionized plasma the electron number density is given by $N_{\mathrm{e}}=\sum_{Z} Z N_{Z}$. Together, the energy loss due to emission of e-n and e-e bremsstrahlung takes the form

$$
\begin{aligned}
-\frac{\mathrm{d} E}{\mathrm{~d} t}= & \alpha r_{0}^{2} m c^{3} S \frac{8 \epsilon^{2}}{\epsilon^{2}+p^{2}}\left\{\epsilon \ln (\epsilon+p)-\frac{p}{3}+\frac{p^{3}}{\epsilon^{6}}\left(\frac{2}{9} \epsilon^{2}-\frac{19}{675} \epsilon p^{2}\right.\right. \\
& \left.\left.-0.06 p^{4} / \epsilon\right)\right\}+\left\{\sum_{Z} Z N_{Z}\right\} m c^{3}(p / \epsilon)(\epsilon-1) \Phi_{\mathrm{rad}}^{\mathrm{ee}}(\epsilon) .
\end{aligned}
$$

The contribution of e-e bremsstrahlung increases rapidly with increasing electron energy $E$. For $E>100 \mathrm{keV}$ the contribution exceeds $10 \%$ of the total bremsstrahlung losses, and for $E>$ $1 \mathrm{MeV}$ its share is more than one third.

It is interesting to compare the radiative energy loss with the loss by collisions which dominate at lower energies. Considering a completely ionized plasma, where no ionization losses occur, the latter is given by losses in electron-electron collisions,

$\frac{\mathrm{d} E}{\mathrm{~d} t}=-4 \pi r_{0}^{2} m c^{3} N_{\mathrm{e}}(\epsilon / p) \ln \Lambda_{\mathrm{ee}}$

The Coulomb logarithm for $v / c=p / \epsilon \gg \alpha$ was given by Gould (1974),

$$
\begin{aligned}
\ln \Lambda_{\mathrm{ee}} & \approx \ln \left(\frac{m v^{2}}{2^{3 / 2} \hbar \omega_{\mathrm{p}}}\right)+\frac{1}{2}=\ln \left\{\frac{p^{2} c^{2}}{\epsilon^{2} \hbar}\left(\frac{\epsilon_{0} m^{3}}{8 e^{2} N_{\mathrm{e}}}\right)^{1 / 2}\right\}+\frac{1}{2} \\
& \approx 36.62+2 \ln (p / \epsilon)-\frac{1}{2} \ln N_{\mathrm{e}}
\end{aligned}
$$

where $\omega_{\mathrm{p}}$ is the plasma frequency, $e$ the electron charge, and $\epsilon_{0}$ the permittivity of vacuum. In the last line of Eq. (11) the electron number density $N_{\mathrm{e}}$ has to be given in units of $\mathrm{cm}^{-3}$. At relativistic energies, where $p / \epsilon \approx 1$, the energy loss rate Eq. (11) is virtually constant. 
Figure 1 shows in a doubly logarithmic scale the bremsstrahlung energy loss rate according to Eq. (9) and the approximation of Blumenthal \& Gould (1970), compared with the energy loss rate by electron-electron collisions. As was to be expected, the approximation is poor at low electron energies $E$. But even in the $\mathrm{MeV}$ energy range there are considerable differences between the accurate calculation (Eq. (9)) and the approximation. Only at high energies $E>80 \mathrm{MeV}$ the two curves converge. At nonrelativistic and mildly relativistic energies the loss rate by collisions exceeds the bremsstrahlung loss rate by several orders of magnitude. The bremsstrahlung loss rate dominates only at $E>512 \mathrm{MeV}$. Here the approximation of Blumenthal \& Gould (1970) is accurate.

\section{References}

Bă̌er, V. N., Fadin, V. S., \& Khoze, V. A. 1967, JETP, 24, 760 (1966, Zh. Eksp. Teor. Fiz., 51, 1135)

Bethe, H., \& Heitler, W. 1934, Proc. Roy. Soc. London A, 146,83

Blumenthal, G. R., \& Gould, R. J. 1970, Rev. Mod. Phys., 42,237

Gould, R. J. 1974, Ann. Phys., 84, 480

Haug, E. 1975, Z. Naturforsch., 30a, 1099

Haug, E. 1997, A\&A, 326, 417

Haug, E. 1998, Sol. Phys., 178, 341

Heitler, W. 1954, The Quantum Theory of Radiation, 3rd edition (Oxford University Press) 